\title{
Nanoporous Structure and Enhanced Thermal Stability of a Polyimide/Single-Walled Carbon Nanotube Composite
}

\author{
Gerard T. Caneba \\ Department of Chemical Engineering and Center for Environmentally Benign \\ Functional Materials, Michigan Technological University, Houghton, MI 49931 \\ Email: caneba@mtu.edu
}

\begin{abstract}
Polymer composites based on polyimides and single-walled carbon nanotubes are of interest in space and aerospace industries, due to inherent thermal stabilities as thermal/electrical conductivities of the components. Unfortunately, it is very difficult to disperse the carbon nanotubes (CNTs) into the polyimide matrix. This work attempts this by predispersion of the CNTs in the precursor reactive materials, and then polymerization is carried out with the CNTs in place. When this is done, some unusual behavior has been observed, such as the formation of nanoporous composite structure and enhanced thermal stability of the products.
\end{abstract}

Keywords: Polyimide, Single-walled Carbon Nanotubes, HiPco, nanoporous structure, thermally stable polymer composite

\section{INTRODUCTION}

High performance polymers, such as polyimides, epoxies, poly(tertrafluoroethylene), polycarbonate, etc. are being used in aerospace and space systems. Carbon nanotubes are of interest as lightweight materials with enhanced mechanical, thermal, and electrical properties. For example, their continuous fibers have been shown to be stronger per weight compared to stainless steel (up to 100 times stronger) and Kevlar (14 times stronger). Also, they can posses 
electrical and thermal conductivities better than Copper. Within a polymeric matrix, thermal diffusivities can be at least 30 times that of the neat polymer. It is also reasonable to expect that layers of carbon nanotubes can act as excellent diffusion barrier materials, based on tight packing of fibers in the nanoscale. It is therefore the aim of this proposed program to formulate composites of various carbon nanotubes with high performance polymers that will possess enhanced mechanical, electrical and thermal conductivity properties. At the same time these composites could possess special surface characteristics, such as radiation protection and monitoring barriers, mass diffusion barriers, tribo-mechanical properties, etc.

In this work, we introduce a procedure to produce polyimide/single walled carbon nanotube composite as nanoporous materials, which exhibits enhanced thermal stability properties. The polyimide we produced came from the condensation polymerization reaction of dianhydrides and a diamine. An intermediate product, poly(amic acid), was formed. Then, imidization was done by water removal from the intermediate.

\section{EXPERIMENTAL}

The dianhydride (Pyromellitic dianhydride, PMDA) and diamine (3,3'-Oxydianiline, ODA) were dried under vacuum overnight at $110^{\circ} \mathrm{C}$. Then, $50 \mathrm{~g}$ of a $0.1 \mathrm{wt} \%$ solution of HiPco in NMP was prepared by adding $0.05 \mathrm{~g}$ of HiPco to $50 \mathrm{~g}$ of NMP in a capped $70 \mathrm{ml}$ test tube. It was best to use conical caps for these test tubes because the NMP tends to dissolve the glue that hold in the foam seal on the non-conical caps. Once the $0.1 w t \%$ HiPco/NMP solution was made up it needs to be sonicated. Before sonication, the caps on the tubes were tightly sealed, since the vibrations from sonication can cause a loose cap to come off. The HiPco/NMP solution was sonicated until nanotubes appeared to be uniformly distributed throughout the NMP. After sonication was complete, the dried PMDA was added to the HiPco/NMP solution and the sonication step was repeated. The amount of PMDA resulted in $1 \mathrm{wt} \% \mathrm{HiPco}$ in the polyimide. While sonication was occurring the ODA was weighed out in a 1:1 mole ratio with the PMDA in the HiPco/NMP solution. The ODA was placed in a $125 \mathrm{ml}$ Erlenmeyer flask with a magnetic stirrer. The PMDA/HiPco/NMP solution was added into the $125 \mathrm{ml}$ flask with the ODA, and mixed overnight to produce the poly(amic acid). Imidization was done the following day by adding in pyridine and acetic anhydride, each at 2:1 mole ratio with the ODA or PMDA. Products were obtained after $24 \mathrm{hrs}$ as gel materials, from which solvents and unreacted material were repeatedly extracted using 190-proof ethanol. Solids were air-dried at room temperature for at least $24 \mathrm{hrs}$ and then vacuum-dried at $\sim 110^{\circ} \mathrm{C}$ overnight.

\section{RESULTS AND DISCUSSION}

Three types of dried bulk products were obtained: (a) HiPco-PI with 0.5 wt \% HiPco; (b) HiPcoPI with 1 wt \% HiPco; and, (c) Pure PI. The HiPco-PI products are black porous materials 
(Figure 1) and the pure PI product is a brown dense solid (Figure 2). Both HiPco-PI products have open-cell structures with about 20-50 nm uniform cells (Figure 3).

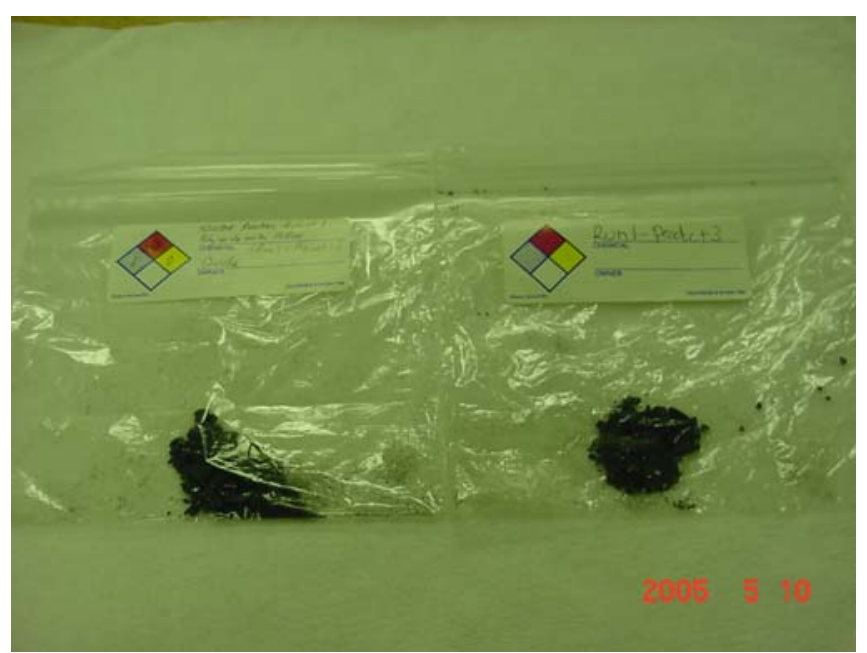

Figure 1. Picture of porous solid materials from PI/HiPco that were obtained from a stoichiometric reaction of 3,3'-ODA and PMDA.

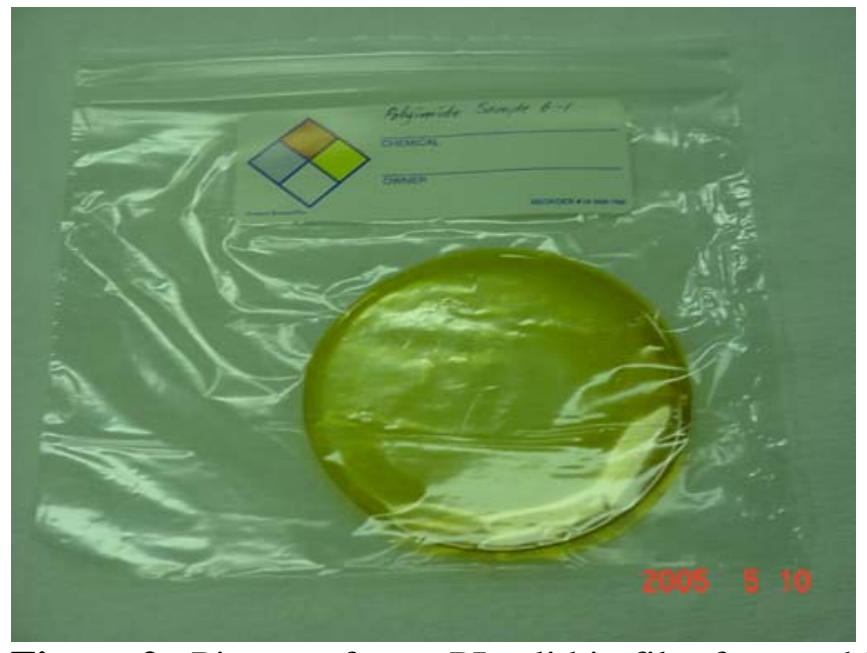

Figure 2. Picture of pure PI solid in film form, which indicates a dense structure. 


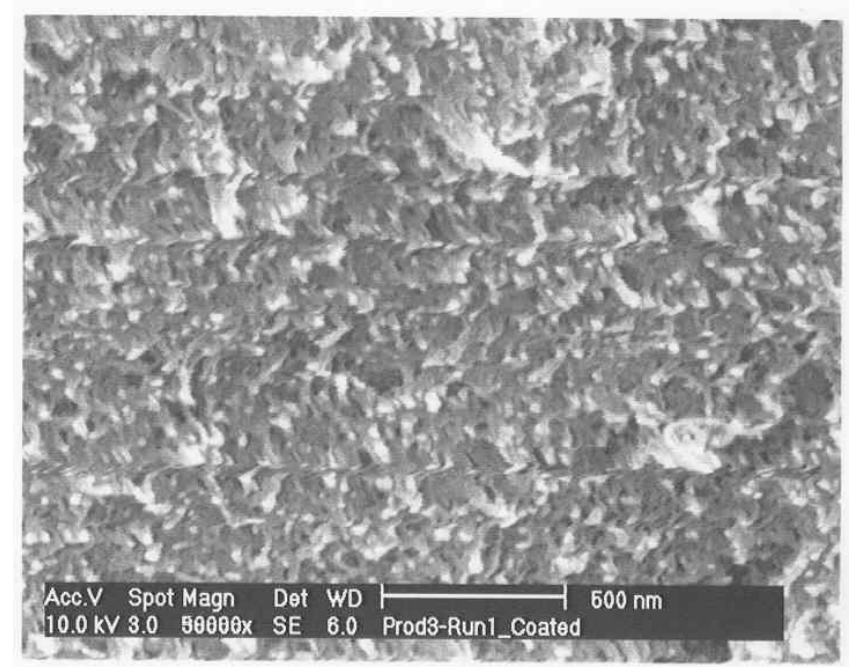

Figure 3. SEM of open cell structure of bulk PI product with $0.5 \mathrm{wt} \%$ HiPco, showing 20-50 nm cells. The PI was obtained from a stoichiometric reaction of 3,3'-ODA and PMDA.

Densities seem to be quite low, and these solids exhibited microcracked domains of 100-500 microns (Figures 4 and 5).

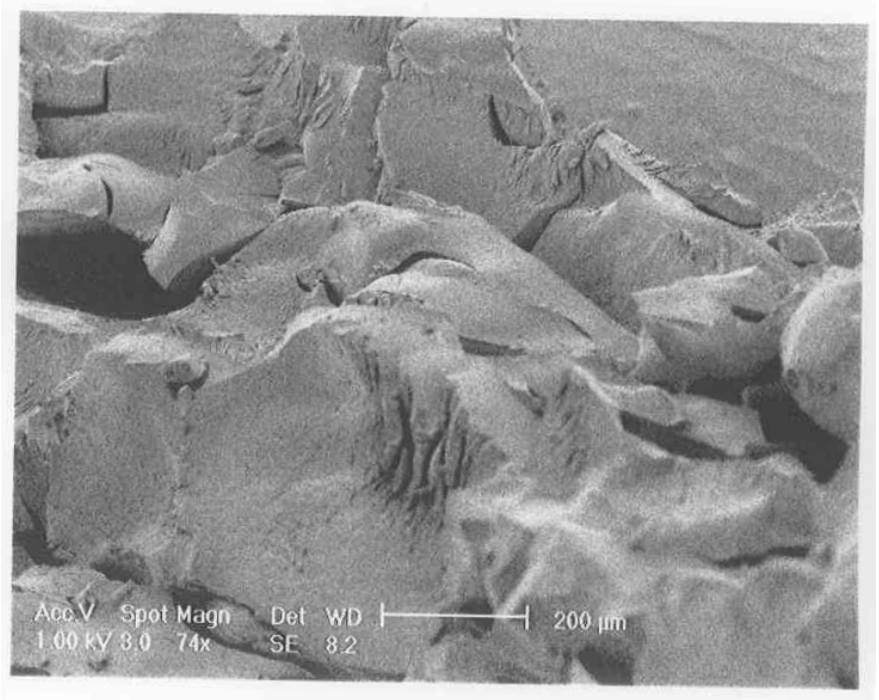

Figure 4. SEM of fractured surfaces of bulk PI with 0.5 wt \% HiPco, showing 100-200 micron microcracked domains. The PI was obtained from a stoichiometric reaction of 3,3'-ODA and PMDA. 


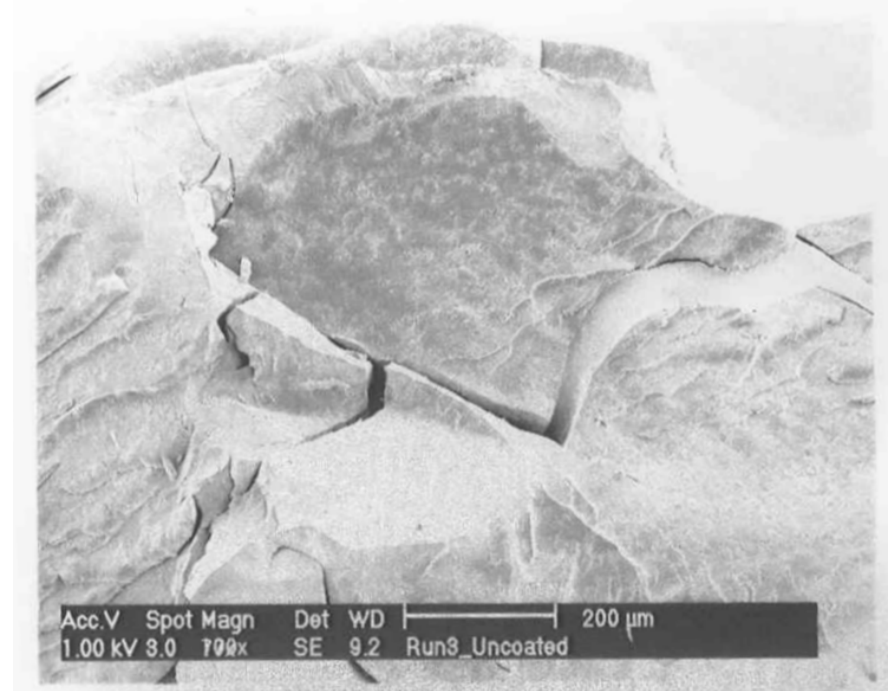

Figure 5. SEM of fractured surfaces of bulk PI with 1 wt \% HiPco, showing 100-500 micron microcracked domains. Also shown on the surfaces is a root structure made up of HiPco bundles. The PI was obtained from a stoichiometric reaction of 3,3'-ODA and PMDA.

This means that 100-500 micron powders can easily be obtained from both HiPco-PI nanoporous materials. This is confirmed after the HiPco-PI porous solid was placed in m-Cresol, which resulted in the breaking up of the solid into 100-500 micon particles (Figure 6). For the 0.5 wt \% HiPco material, the HiPco bundles are not visible even under 50,000X magnification on an SEM. For the $1 \mathrm{wt} \%$ HiPco sample, root structures are evident (Figure 5), especially at a magnification of 2,000X (Figure 7).

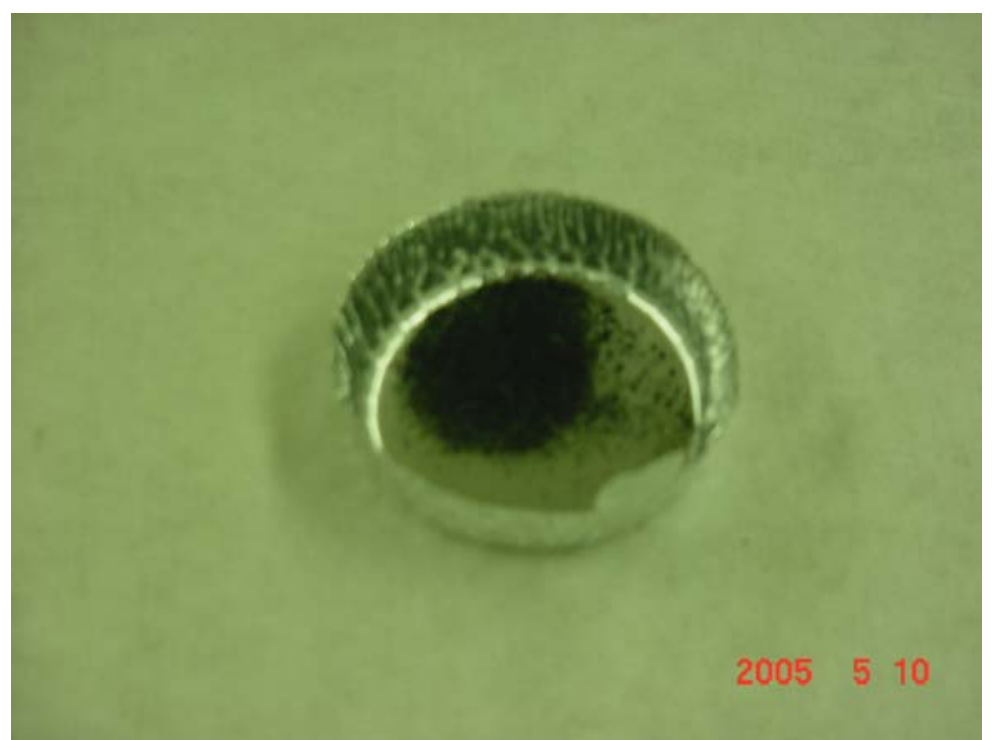

Figure 6. PI/HiPco 100-500 micron particles after adding m-cresol into porous solid in Figure 1 and drying the resulting suspension. 


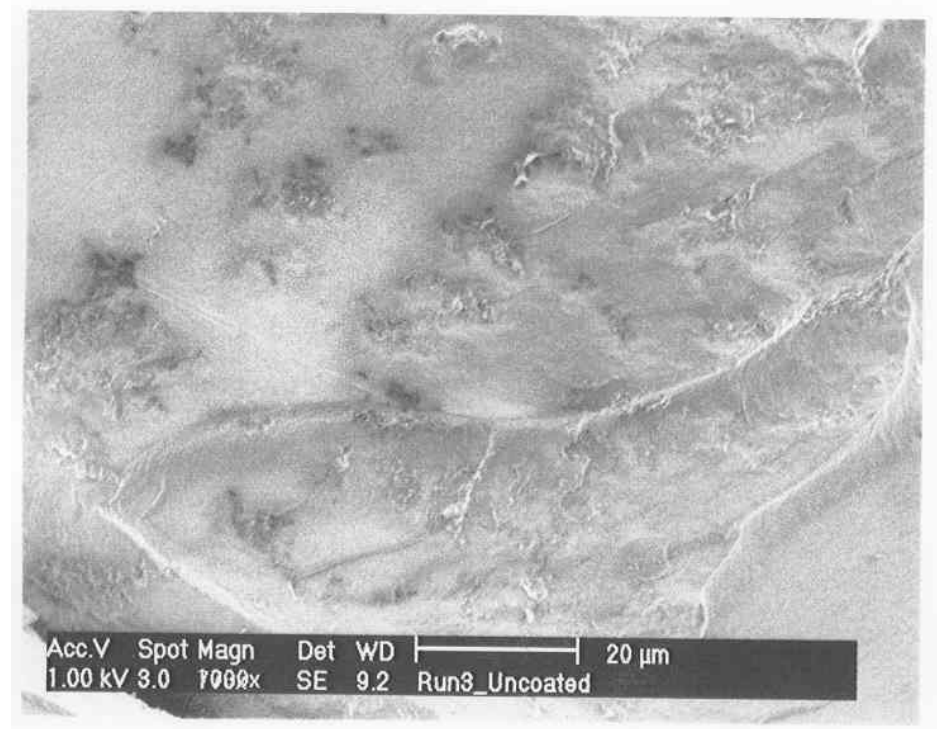

Figure 7. SEM of a fractured surface of bulk PI with $1 \mathrm{wt} \% \mathrm{HiPco}$, showing a root structure made up of HiPco bundles at a magnification of 1000X. The PI was obtained from a stoichiometric reaction of 3,3'-ODA and PMDA.

Protruding structures from these root patterns are "thorns" that are about $600 \mathrm{~nm}$ long and 100 nm wide (Figure 8); their surfaces seem to indicate aligned nanotube bundles (Figure 9).

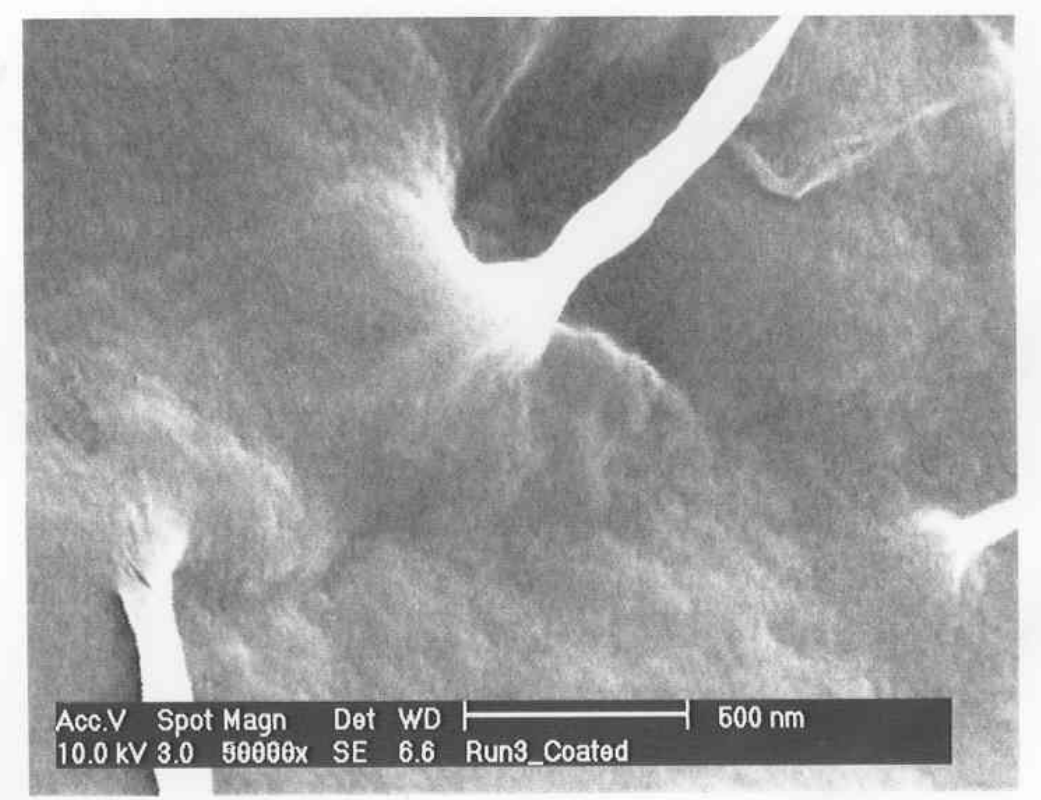

Figure 8. SEM of a fractured surface of bulk PI with 1 wt \% HiPco, showing "thorns" emanating from the root structure in Figures 4 and 5. The PI was obtained from a stoichiometric reaction of 3,3'-ODA and PMDA. 


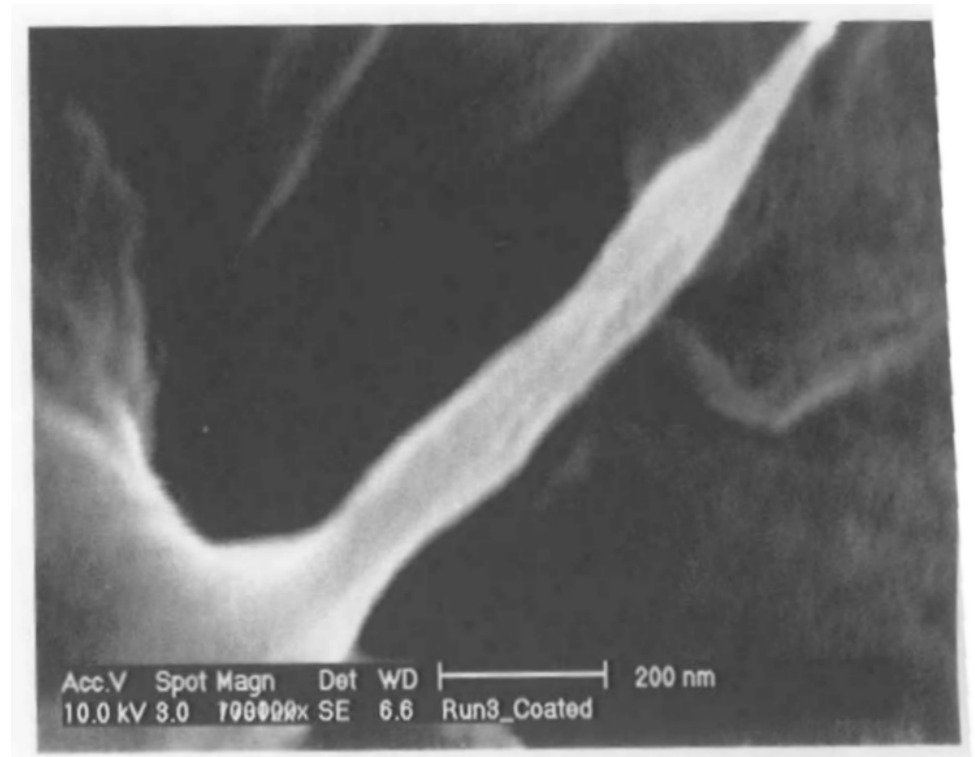

Figure 9. SEM of a "thorn" from a fractured surface of bulk PI with 1 wt \% HiPco. The PI was obtained from a stoichiometric reaction of 3,3'-ODA and PMDA.

The base of a protrusion also shows nanotube bundles that serve as nanoanchors. Why are the $\mathrm{HiPco} / \mathrm{PI}$ materials nanoporous while the pure PI is a dense material? It is believed that the HiPco structure served as a rheology modifier for the reactive system, as also evidenced by observation of increased viscosities. The gel of the PI with small molecule components could not turn into dense solids while drying because of the presence of the nanotube bundles and fibers.

Thermal properties of the nanoporous HiPco-PI composites are quite interesting. TGA analysis showed the HiPco burn-off temperature of about $500^{\circ} \mathrm{C}$ in air (Figures 10 and 11). PI burn-off occurred at $500^{\circ} \mathrm{C}$ and at $615^{\circ} \mathrm{C}$ and $800^{\circ} \mathrm{C}$ for 0.5 and $1 \mathrm{wt} \% \mathrm{HiPco}$, respectively. In nitrogen gas, complete gasification of PI was not observed up to $1000^{\circ} \mathrm{C}$ (Figures 12 and 13). About 50\% of material still remained. Also, in air, the composite with $1 \mathrm{wt} \% \mathrm{HiPco}$ is more thermally stable than the one with only $0.5 \mathrm{wt} \%$ HiPco. DSC analysis of the $1 \mathrm{wt} \%$ HiPco composite indicates no distinct second-order transition until HiPco burn-off. This means that the polyimide is unprocessable and cannot be remelted. It should be noted that typical onset of decomposition temperatures for polyimides based on polypyromellitides are obtained based on the diamines used. ${ }^{1}$ If the diamine is based on a hexamethylene group, the onset of decomposition occurs at $285^{\circ} \mathrm{C}$ in air and $370^{\circ} \mathrm{C}$ in Nitrogen gas. For a diamine based on a 1,3-benzene group, the onset of decomposition occurs at $318^{\circ} \mathrm{C}$ in air and $530^{\circ} \mathrm{C}$ in Nitrogen gas. Nanofoams made from polyimides based on an oxydianiline-pyromellitic dianhydride through grafting with labile polypropylene glycol oligomers have also been found to decompose at around $600^{\circ} \mathrm{C}{ }^{2}$. These data indicate that our polyimide-carbon nanotube composites seem to exhibit self-stabilizing thermal behavior, because as shown in Figures 12 and 13, decomposition does not occur in Nitrogen even above $1,000^{\circ} \mathrm{C}$. 
It is evident that a number of unusual structural and thermal behavior has been observed during the formation of PI/HiPco composites from the in situ CNT procedure used in this work. More experimental efforts will be needed in order to explain these results.

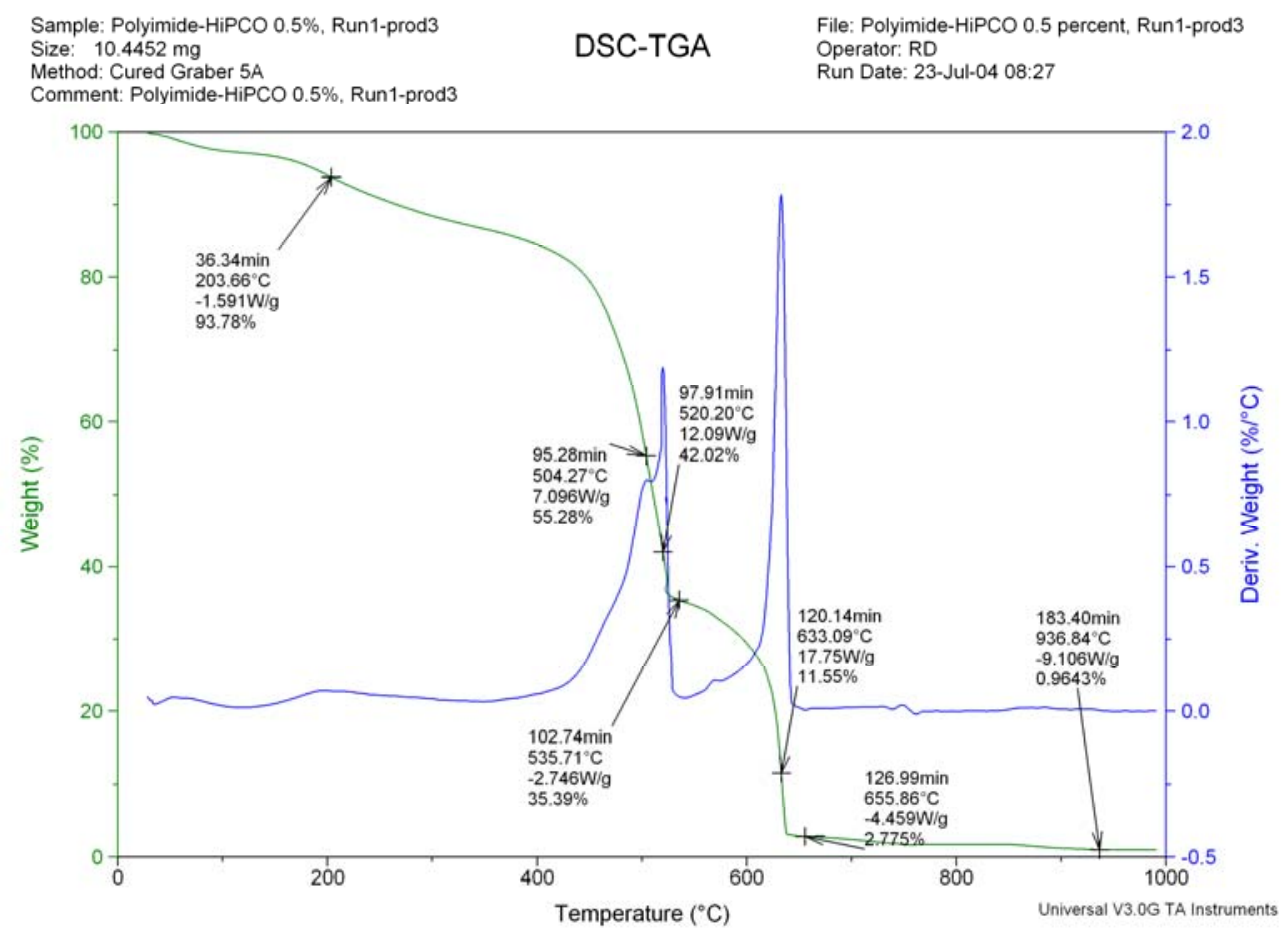

Figure 10. DSC-TGA results of $0.5 \mathrm{wt} \% \mathrm{HiPco}$ in PI in air.

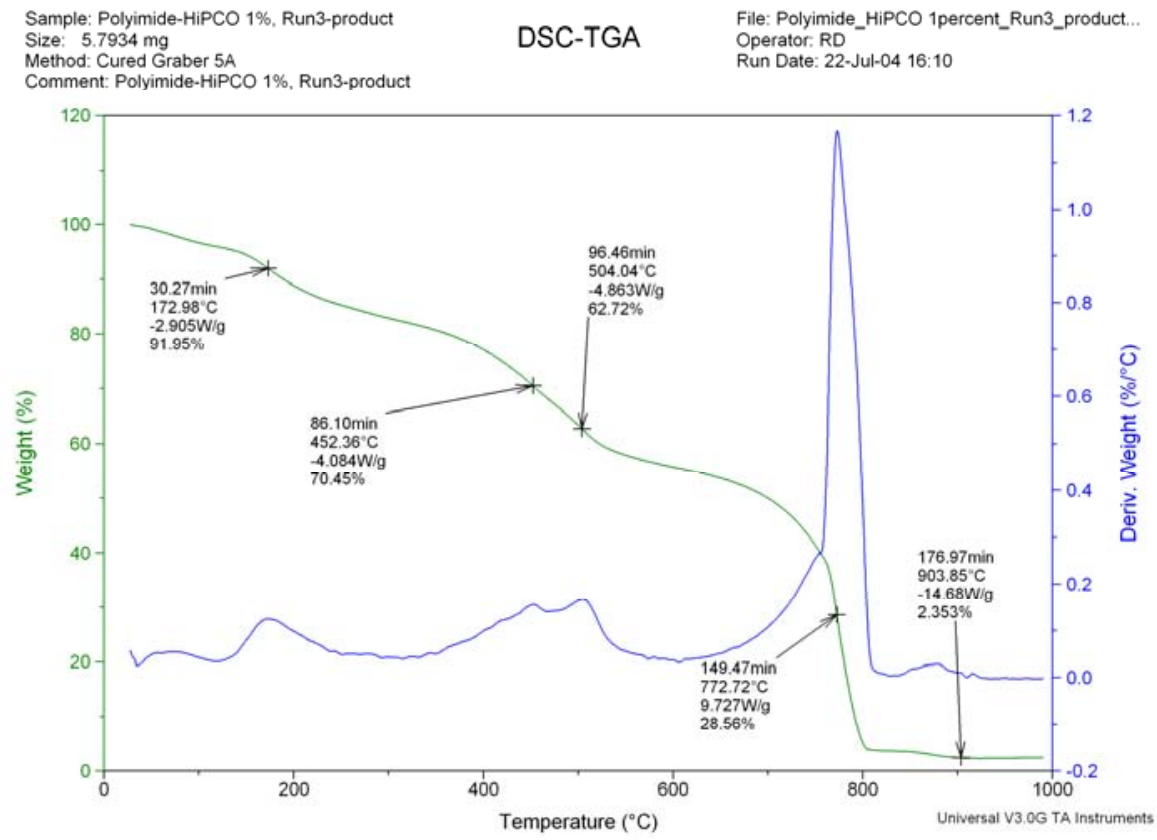

Figure 11. DSC-TGA results of $1 \mathrm{wt} \% \mathrm{HiPco}$ in PI in air. 


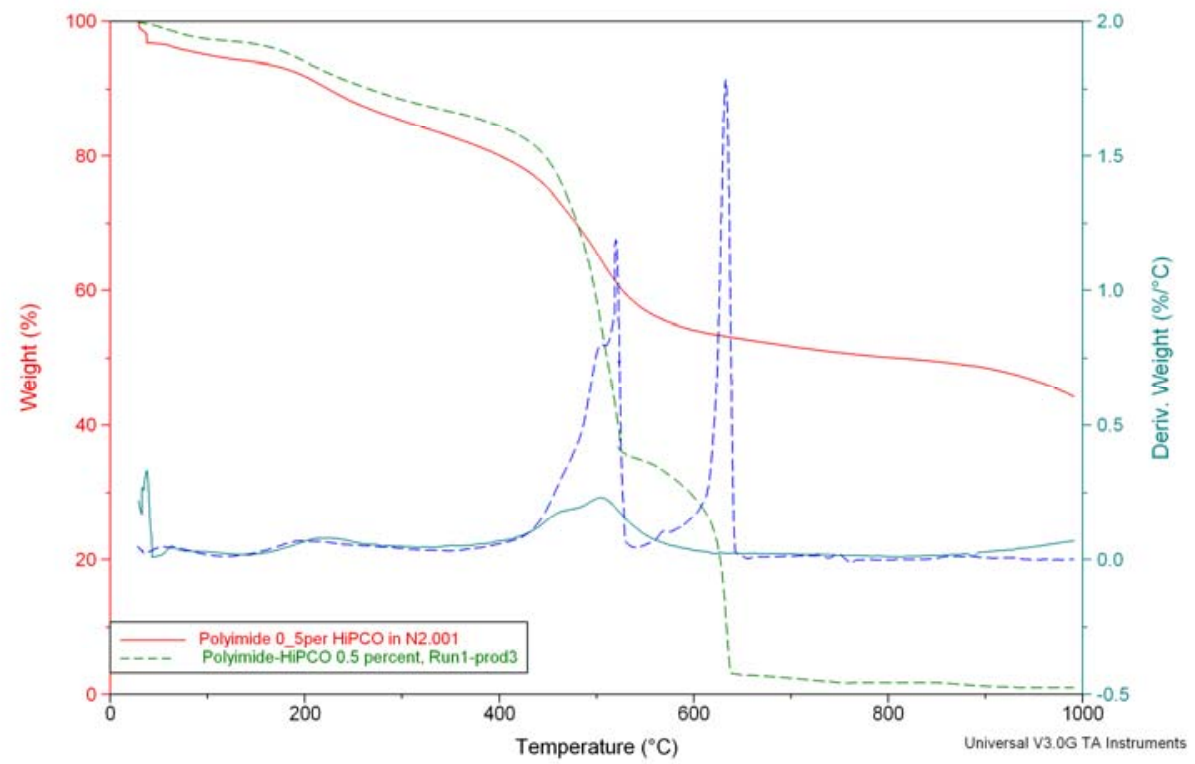

Figure 12. DSC-TGA comparison results of $0.5 \mathrm{wt} \%$ HiPco in PI in air and under nitrogen gas atmosphere.

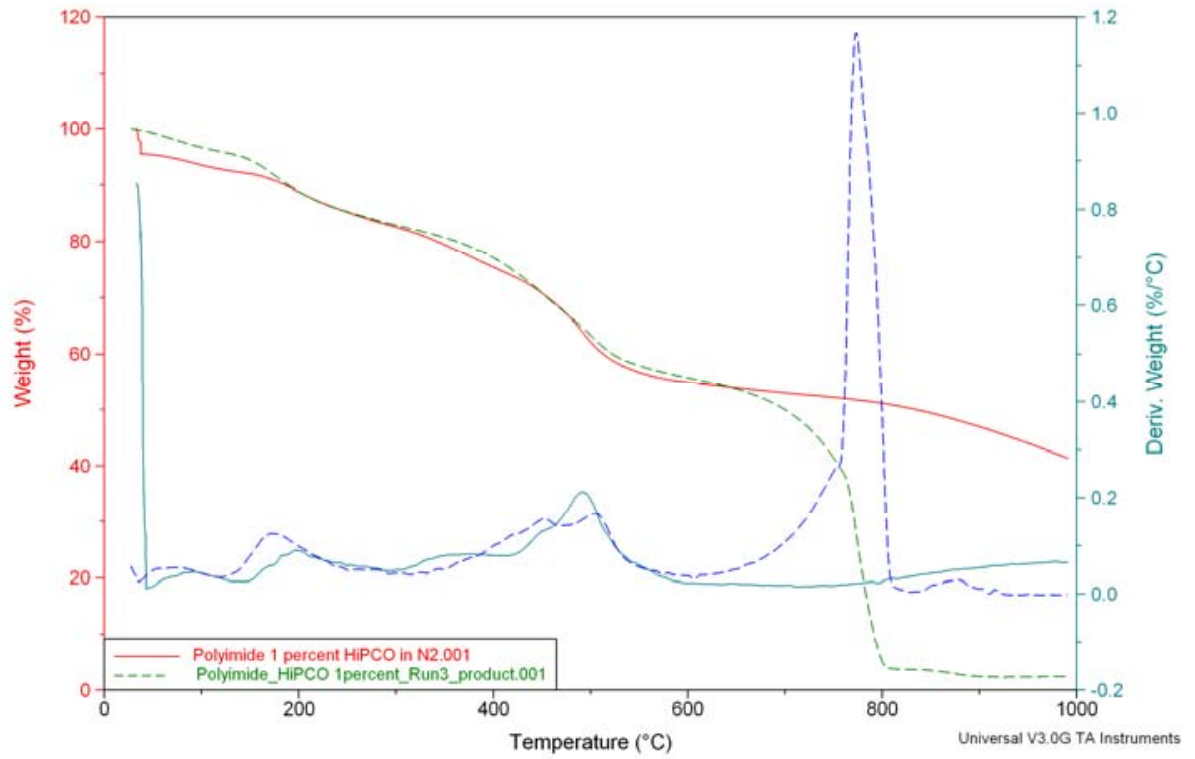

Figure 13. DSC-TGA comparison results of $1 \mathrm{wt} \%$ HiPco in PI in air and under nitrogen gas atmosphere. 


\section{CONCLUSION}

The physical predispersion of HiPco type single-walled carbon nanotubes in precursor fluids of polyimide formation not only allowed the dispersion of the CNT into the polymer, but also resulted in nanoporous composite structure and thermally more stable materials.

\section{Acknowledgements}

The author acknowledges the Carbon Nanotubes Group of the Structural Engineering Division of NASA-Johnson Space Center for various contributions to this research effort, including the supply of HiPco, SEM work, and some financial assistance. Acknowledgement is also provided to Dr. Patricia Heiden of the Department of Chemistry for pointers on polyimide synthesis. Finally, the author acknowledges the Michigan Technological University Center for Environmentally Benign Functional Materials (CEBFM) for providing the setting for manuscript preparation and continuing efforts from this work.

\section{References}

1. Verbicky, Jr., J.R., "Polyimides”, in: Concise Encyclopedia of Polymer Science and Engineering, J.I. Kroschwitz (Ed.), John Wiley and Sons, New York, 1990, pp.826-828.

2. Han, S.H., Do, J.-S., Abdul Kader, M., Lee, J.-H., Lee, M.-H., and Nah, C., "Preparation and Characterization of a Polyimide Nanofoam through grafting of labile Poly(propylene glycol) Oligomers”, Polymers for Advanced Technologies, 15(7), 370-376 (2004). 